\title{
Climate Feedback: Wissenschaft kommentiert Journalismus und entwickelt Mehrsystemkompetenz
}

\author{
Stefanie Walter · Janne Görlach • Michael Brüggemann
}

Online publiziert: 1 . Oktober 2020

(C) Der/die Autor(en) 2020, korrigierte Publikation 2020

Zusammenfassung Wissenschaft und Journalismus beruhen auf unterschiedlichen Logiken. Aus Sicht der Systemtheorie überrascht es also nicht, wenn WissenschaftlerInnen Wissenschaftsjournalismus kritisieren. Gleichzeitig wird aber auch eine Medialisierung von Wissenschaft postuliert. Demnach würde sich Wissenschaft zunehmend an Medienlogiken orientieren. Diese Studie prüft explorativ, welche Kriterien WissenschaftlerInnen bei der Beurteilung journalistischer Artikel heranziehen und welche Aspekte sie loben und kritisieren. Dazu werten wir die Kommentare auf dem Blog „Climate Feedback“ qualitativ inhaltsanalytisch aus. Induktiv werden zunächst die angelegten Evaluationskriterien der WissenschaftlerInnen kategorisiert und dann den Überkategorien ,journalistische Vermittlungsleistung“ oder „wissenschaftliche Informationsleistung" zugeordnet. Unsere Ergebnisse, basierend auf 82 Blogeinträgen und den Kommentaren von 184 WissenschaftlerInnen im Zeitraum von 2015 bis 2017, zeigen, dass sich die WissenschaftlerInnen intensiv und sogar häufiger mit Aspekten der journalistischen als der wissenschaftlichen Leistung beschäftigen. Sie sehen die journalistischen Kriterien eher als erfüllt an, während sie das Fehlen wissenschaftlicher Standards kritisieren. Die beteiligten WissenschaftlerInnen kombinieren die Kommunikationsnormen beider Systeme. Für den Ansatz der Medialisierung von Wissenschaft ergibt sich der Befund, dass die Diffusion von

\footnotetext{
S. Walter, Ph.D. $(\bowtie)$

ZeMKI, Zentrum für Medien-, Kommunikations- und Informationsforschung, Universität Bremen, Linzer Str. 4, 28359 Bremen, Deutschland

E-Mail: Stefanie.Walter@uni-bremen.de

J. Görlach, M.A.

Universität Hamburg, Allende-Platz 1, 20146 Hamburg, Deutschland

E-Mail: janne.goerlach@gmx.de

Prof. Dr. M. Brüggemann

Journalistik und Kommunikationswissenschaft, Universität Hamburg,

Allende-Platz 1, 20146 Hamburg, Deutschland

E-Mail: Michael.Brueggemann@uni-hamburg.de
} 
Medienlogiken keineswegs zur Aufgabe von Logiken der Wissenschaft führen muss, sondern dass kompetente Akteure an der Schnittstelle zwischen Journalismus und Wissenschaft Mehrsystemkompetenz erwerben und anwenden können.

Schlüsselwörter Wissenschaftsjournalismus - Wissenschaftskommunikation · Blogs $\cdot$ Klimawandel $\cdot$ Inhaltsanalyse $\cdot$ Medialisierung

\section{Climate feedback: Science comments on journalism and develops multi- system competence}

Abstract Science and journalism function according to different logics. From the perspective of systems theory, it is therefore not surprising that scientists see science journalism critically. At the same time, a medialization of science has been observed, meaning that science increasingly takes media logics into account. This exploratory study examines which criteria scientists apply when evaluating journalistic articles and which aspects they praise and criticize. To this end, a qualitative content analysis of comments on the blog Climate Feedback was conducted. We first inductively derived the evaluation criteria applied by the scientists commenting on the news stories and then assigned them to the superordinate categories "journalistic brokerage" and "scientific information performance". The empirical analysis is based on 82 blog entries and the comments by 184 scientists published between 2015 and 2017.

With regard to scientific information performance, we identify seven quality criteria that scientists apply. These are strongly oriented towards the principles that are relevant for the scientists' own work: Evidence, comprehensiveness, conformity with the scientific state of the art, the proportionality and temporary nature of scientific findings, as well as a logical reasoning. They also demand the recognition of scientific credibility.

Scientists evaluate the journalistic performance based on five criteria: Adherence to facts, mediation, the professional approach of the journalist, the contextualization of information, and the diversity of positions and sources. Here, scientists evaluate core competencies of journalists, which are determined by journalistic norms and values. By doing so, the scientists transcend their own system's boundaries: Not only do they apply criteria relevant to their own system, but they are even more frequently concerned with the journalistic performance.

The blog Climate Feedback can be seen as a hybrid organization at the intersection of two systems. It aims to evaluate the quality of the news media coverage by applying the scientific procedure of peer review. In order to enhance the quality of the news media coverage, an internal quality assurance measure is applied to a non-scientific system. Although systems theory takes into account that journalists must be able to identify the relevance of events from the perspective of different systems and that individual actors may well switch between systems depending on the context, the results not only show that competent individuals can switch from one system to another, but that they can simultaneously apply the logics of two different systems. We thus observe the co-existence of quality criteria from the two systems, not a blurring of boundaries between them. 
The study also provides insights for the medialization approach: The existence of the blog itself can be seen as evidence of medialization. It is the blog's objective to evaluate influential media reports on climate change. This is an indicator that the news media coverage is considered relevant for fulfilling the scientific system's goal. The comments analyzed, however, do not show a one-sided adoption of media logics by scientists. Instead, competent actors at the interface between journalism and science seem to acquire and apply multi-system competence.

Keywords Science journalism - Science communication $\cdot$ Blogs $\cdot$ Climate change Content analysis $\cdot$ Medialization

\section{Einleitung}

Der Klimawandel ist ein langfristiges, komplexes und globales Phänomen, das unmittelbar nicht erfahrbar ist. Gleichzeitig ist er eine der großen Herausforderungen dieser Zeit. Wissenschaftliche Erkenntnisse zum Klimawandel werden systematisch in Zweifel gezogen (vgl. z.B. Dunlap und McCright 2015). WissenschaftlerInnen sehen sich mit dem Vorwurf von ,fake science“ konfrontiert. Darum sind gerade bei diesem Thema Journalismus und Wissenschaftskommunikation so wichtig (vgl. Brüggemann et al. 2018; Neverla und Schäfer 2012).

Was guter Wissenschaftsjournalismus ist und sein soll, ist in digitalen Medienwelten mehr denn je ein Gegenstand öffentlicher Aushandlung, an der sich mit UserKommentaren, Blogbeiträgen, Weiterleitungen und Bewertungen auf Netzwerken jeder beteiligen kann - auch die Wissenschaft. Neue digitale Kommunikationskanäle bieten WissenschaftlerInnen ein Forum, um ihre Kritik am Wissenschaftsjournalismus öffentlich zu äußern. Ein bisher einmaliges Unterfangen wissenschaftlicher Medienkritik und Qualitätssicherung ist das im Jahr 2015 gestartete Blog „Climate Feedback", in dem WissenschaftlerInnen journalistische Beiträge detailliert kommentieren und annotieren. Die Beiträge bieten Einblick in die Bewertungsmaßstäbe, die die beteiligten WissenschaftlerInnen an den Wissenschaftsjournalismus anlegen.

Trotz der Schlüsselrolle des Journalismus bei der Informationsvermittlung über den Klimawandel gibt es bisher relativ wenige Untersuchungen darüber, wie KlimawissenschaftlerInnen die mediale Darstellung der Klimaforschung bewerten (vgl. Farnsworth und Lichter 2012, S. 436). Kommunikationswissenschaftlich relevant ist dieses Forschungsinteresse im Hinblick auf die These der Medialisierung von Wissenschaft (vgl. Weingart 2001) und die Untersuchung von Akteurshandeln an der Schnittstelle der Systeme Journalismus und Wissenschaft unter den Bedingungen digitaler Netzwerköffentlichkeit.

Die Bereitstellung und Vermittlung von aktuellen Informationen und Themen für den öffentlichen Diskurs ist die zentrale Funktion des Wissenschaftsjournalismus (vgl. Blöbaum 2017; Kohring 1997). Dabei müssen wissenschaftliche Ergebnisse so präsentiert werden, dass sie für ein Laienpublikum verständlich sind. Dies kann zu Konflikten und Missverständnissen zwischen WissenschaftlerInnen und JournalistInnen führen. Medienwandel und vor allem das Internet verändern auch die Normen der Wissenschaftskommunikation und die Beziehung zwischen Wissenschaft und 
Journalismus (vgl. Brüggemann et al. 2020). Digitale Kommunikationskanäle erleichtern es WissenschaftlerInnen, direkt mit JournalistInnen in Kontakt zu treten (vgl. Peters et al. 2014; Walter et al. 2019), sie als Gatekeeper zu umgehen (vgl. Walter und Brüggemann 2020) oder öffentlich Kritik am Wissenschaftsjournalismus zu äußern.

Ziel dieser Studie ist es, zu analysieren, wie WissenschaftlerInnen die Medienberichterstattung über Klimawandel bewerten und welche Maßstäbe sie hierbei zugrunde legen. Die Rolle von WissenschaftlerInnen bei der Bewertung von journalistischen Inhalten wird mit einer qualitativen Inhaltsanalyse überprüft. Grundlage sind 82 Blogposts und den Kommentare von 184 WissenschaftlerInnen, die zwischen dem 29.01.2015 und dem 04.12.2017 auf dem Medienwatchblog Climate Feedback veröffentlich wurden. Die Ergebnisse zeigen, dass sich WissenschaftlerInnen stark an Bewertungskriterien aus der Wissenschaft orientieren, aber auch originär journalistische Kriterien (z.B. verständliche Darstellung, sprachlicher Ausdruck usw.) heranziehen. Die Ergebnisse dieser Studie sind von Bedeutung, da sie einerseits pauschale Annahmen einer Kolonisierung der Wissenschaft durch mediale Logiken widerlegen. Umgekehrt verharren WissenschaftlerInnen auch nicht in der eigenen Systemlogik. Vielmehr erwerben sie Mehrsystemkompetenz.

\section{Qualität von Wissenschaftsjournalismus und Klimaberichterstattung}

Für Wissenschaftsjournalismus gelten andere Kriterien als etwa für die politische Berichterstattung. Die Güte von Wissenschaftsjournalismus wird oft an der Nähe zur zugrunde liegenden wissenschaftlichen Information gemessen (vgl. Kohring 1997, S. 92-93). Häufige Kritikpunkte am Wissenschaftsjournalismus beinhalten eine zu starke Vereinfachung von Wissenschaft, mangelnde Akkuratesse, Sensationalismus, Panikmache, Personalisierung, Verzerrungen und die unverhältnismäßige Berücksichtigung von Außenseitermeinungen (vgl. Dudo 2015, S. 762). Dennoch ist Wissenschaftsjournalismus in erster Linie Journalismus, und so äußern JournalistInnen auch Kritik an der Wissenschaft. Sie werfen WissenschaftlerInnen mangelnde Kooperationsbereitschaft, Arroganz, Egozentrik, lange Reaktionszeiten bei journalistischen Anfragen, Einseitigkeiten und den Anspruch vor, über journalistische Inhalte bestimmen zu wollen (vgl. Dudo 2015, S. 762). Dies deutet auf schwierige Voraussetzungen für eine erfolgreiche Zusammenarbeit hin. Dennoch stellt Wissenschaftskommunikation eine zentrale gesellschaftliche Wissensressource dar und die Wissenschaft ist auf gesellschaftliche Legitimation angewiesen (Schäfer et al. 2015).

Forschung zur Bewertung wissenschaftlicher Berichterstattung durch die Forschenden selbst beruht größtenteils auf Befragungen von WissenschaftlerInnen (z. B. Post 2016). Ergebnisse zeigen, dass die Mehrheit der KlimawissenschaftlerInnen glaubt, die Medienberichterstattung habe einen sehr großen Einfluss auf die öffentliche Wahrnehmung von Klimawandel (vgl. Bray und von Storch 2007). KlimawissenschaftlerInnen beklagen eine verzerrte mediale Darstellung zwischen Übertreibung und Bagatellisierung und eine irreführende Abbildung von Wissenschaft, sodass es für Laien kaum möglich sei, sich ein ,,sachlich korrektes Bild vom Wissensstand in der Klimaforschung zu machen“ (Rahmstorf und Schellnhuber 2007, S. 89; siehe 
auch vgl. Bray und von Storch 2007). Dies wird auf mangelnde Kompetenz und Interessenseinflüsse zurückgeführt (vgl. Rahmstorf und Schellnhuber 2007, S. 89).

Auch die Auswahl der ExpertInnen, auf die in der journalistischen Berichterstattung zurückgegriffen wird, kann zu Verzerrungen führen: Neben reputierten Forschern suchen JournalistInnen oft nach WissenschaftlerInnen, die dazu bereit sind, meinungshaltige und prägnante Botschaften $\mathrm{zu}$ formulieren (vgl. Lehmkuhl und Leidecker-Sandmann 2019, S. 484). In Befragungen deutscher KlimawissenschaftlerInnen äußerten sich diese kritisch über die vereinfachte mediale Darstellung wissenschaftlicher Themen und die damit einhergehende einseitige Wiedergabe einer vielstimmigen wissenschaftsinternen Debatte (vgl. Post 2016, 2008). Gleichermaßen kritisierten sie einen verzerrten Umgang mit der Aussagekraft von Klimamodellen, die bei eindeutigen Ergebnisdarstellungen tendenziell überschätzt werden (vgl. Post 2008, S. 113).

Daneben befassen sich sogenannte Accuracy-Studien mit der Qualität von Klimaund Wissenschaftsberichterstattung. Farnsworth und Lichter (vgl. 2012. S. 451) stellten in ihrer Untersuchung über die Bewertung journalistischer Beiträge in führenden US-amerikanischen Nachrichten- und Unterhaltungsmedien durch MeteorologInnen und GeophysikerInnen fest, dass lediglich $68 \%$ der Presseberichte über den Klimawandel als ,,somewhat reliable“ bewertet werden. Klimawissenschaftliche Beiträge im Fernsehen werden als noch weitaus weniger verlässlich eingestuft (vgl. Farnsworth und Lichter 2012, S. 445). In der Regel wird hierbei die Sichtweise von WissenschaftlerInnen richtig wiedergegeben, aber durch die Standpunkte von politischen und anderen nicht-wissenschaftlichen Akteuren verzerrt (vgl. Farnsworth und Lichter 2012, S. 438-439). Grundsätzlich lässt sich jedoch festhalten, dass JournalistInnen den wissenschaftlichen Konsens über den anthropogenen Klimawandel teilen und dies auch in ihrer Berichterstattung deutlich wird (vgl. Brüggemann und Engesser 2017).

Insbesondere die Schwierigkeiten bei der Kommunikation von wissenschaftlicher Unsicherheit stellen ein zentrales Problem zwischen Klimawissenschaft und Klimaberichterstattung dar (vgl. Painter 2013): Kalkulierbare Störungen, wie z. B. unterschiedliche Begriffskonnotationen, beeinflussen die Übersetzung von Wissenschaft für die Allgemeinheit ebenso wie die Unsicherheiten, mit denen modellierte Vorhersagen behaftet sind. Zudem wird ein Teil der Klimaberichterstattung von Katastrophenszenarien dominiert.

\section{Die (schwierige) Beziehung von Wissenschaft und Journalismus}

Sowohl Journalismus als auch Wissenschaft können als Funktionssysteme der Gesellschaft angesehen werden (vgl. Blöbaum 1994). Die zentrale Funktion des Journalismus ist die „Herstellung und Bereitstellung von Themen zur öffentlichen Kommunikation“ (Rühl 1980, S. 323; siehe auch Blöbaum 2016). Der ursprünglich von Luhmann (1996) vorgeschlagene binäre Code Information/Nicht-Information, nach dem sich das System der Massenmedien ausrichte, wurde in der deutschen Journalismustheorie weithin kritisiert (vgl. Kohring 1997, S. 236; Scholl und Weischenberg 1998, S. 73). Stattdessen kann Aktualität als Code für Systemzugehörigkeit ange- 
sehen werden, bestehend aus den Kriterien Neuigkeit, Faktizität und Relevanz (vgl. Scholl und Weischenberg 1998, S. 78). Das System Journalismus hat sich ausdifferenziert, zum Beispiel durch die Entwicklung von Ressorts auf Organisationsebene, wie das Wissenschaftsressort (vgl. Blöbaum 2016, S. 155).

Aufgrund von Medienwandel und Veränderungen in gesellschaftlichen Kommunikationsprozessen haben einige eine Entgrenzung des Journalismus attestiert, bei dem es zu einer Auflösung der Systemgrenzen, aber auch der Grenzen zwischen Journalismus und Publikum komme (vgl. im Überblick Loosen 2016).

Die Aufgabe des Wissenschaftssystems ist die Produktion von neuem Wissen und dessen Bereitstellung für die Gesellschaft, wobei Wahrheit der Code ist, mit der die Zugehörigkeit zum Funktionssystem geprüft und festgestellt werden kann (vgl. Krause 2005, S. 50; Franzen et al. 2012, S. 11). Die wissenschaftliche Produktion von Wissen zeichnet sich etwa durch bestimmte Validierungsmethoden, Verlässlichkeit und den Grad an Institutionalisierung aus (vgl. Franzen et al. 2012, S. 8).

Auch das System Wissenschaft ist von gesellschaftlichen Veränderungsprozessen betroffen. Hier lässt sich zunächst einmal die Medialisierung anführen, d.h. die zunehmend engere Kopplung von Wissenschaft und Massenmedien (vgl. Franzen et al. 2012; Weingart 2001). Die These der Medialisierung postuliert zum einen intensivierte Interaktionen zwischen Wissenschaft und Journalismus, und zum anderen Anpassungen und Übernahmen von Medienlogiken seitens der Wissenschaft, die auf eine bessere Sichtbarkeit in der Öffentlichkeit abzielen (vgl. Rödder 2009; Ivanova et al. 2013). Dies gilt insbesondere für die Klimawissenschaft. Empirische Analysen zeigen, dass WissenschaftlerInnen häufig in Kontakt mit JournalistInnen stehen, was Wissenschaft enger an das gesellschaftliche Teilsystem Medien koppelt. Aber nur eine Minderheit der WissenschaftlerInnen (18\%) gibt an, sie berücksichtige mögliches Medieninteresse, wenn sie wissenschaftliche Entscheidungen (z.B. über Forschungsfragen) treffe (vgl. Ivanova et al. 2013).

$\mathrm{Zu}$ einem gewissen Grad sind WissenschaftlerInnen also bereit, sich an die Ansprüche der Medien anzupassen, was aber in der Regel nicht die wissenschaftliche Tätigkeit selbst betrifft. In post-normaler Situationen mit politischem Entscheidungsdruck unter großer Unsicherheit, angesichts des digitalen Medienwandels und weiterer gesellschaftlicher Umwälzungen verändern sich zudem beide Systeme in die gleiche Richtung, so dass nicht nur Spill-Over-Prozesse vom Journalismus auf die Wissenschaft, sondern auch die Stärkung gemeinsamer Normen einer ,post-normalen Wissenschaftskommunikation“" postuliert werden (Brüggemann et al. 2020).

Auch so schon eint Wissenschaft und Journalismus das Ziel der Beschreibung von Tatsachen auf der Grundlage empirischer Recherchemethoden: „Journalism has borrowed its epistemological perspective and ideals from modern science“ (Eide und Kunelius 2012, S. 16). Doch gerade diese Gemeinsamkeit führt zu Irritationen (im umgangssprachlichen und im systemtheoretischen Sinne), wenn Akteure die unterschiedlichen Logiken und Programme beider Systeme nicht durchschauen. Die funktionale Ausdifferenzierung beider Systeme hat zu teilweise inkongruenten Arbeitsweisen von JournalistInnen und WissenschaftlerInnen geführt. Auch die negative Bewertung der Wissenschaftsberichterstattung durch WissenschaftlerInnen lässt sich durch unterschiedliche Methoden und Logiken erklären, die Journalis- 
mus und Wissenschaft als Teilsysteme der Gesellschaft entwickelt haben, um ihren gesellschaftlichen Auftrag zu verfolgen.

JournalistInnen verpflichten sich, der Öffentlichkeit aktuelle und gesellschaftlich relevante Informationen zu liefern, somit definiert und dirigiert Aktualität die Praxis journalistischer Tätigkeit, was zum Teil mit dem Wahrheitsanspruch konkurriert (vgl. Post 2013, S. 47). Die Wissenschaft wiederum verlegt sich auf die Erzeugung von verlässlichem Wissen, mit wissenschaftlichen Methoden und ohne die Bindung an aktuelle Ereignisse (vgl. Post 2013, S. 48).

Eine weitere Komponente, die in der funktionalen Differenzierung beider Systeme hervorsticht, ist die der Qualitätssicherung. Sie folgt im Journalismus keiner allgemeingültigen Regel (z.B. vgl. Meier 2007, S. 225), ,aber es gibt [...] Qualitätskriterien, die in Journalistik wie im Journalismus breite Zustimmung finden“ (Wormer 2017, S. 436). Die Qualitätssicherung in der Wissenschaft wiederum ist durch die Praxis des Peer-Reviews institutionalisiert. Die Diskussion von wissenschaftlichen Ergebnissen, Tatsachenbehauptungen und Interpretationen ist integraler Bestandteil des Wissenschaftsbetriebs (vgl. Post 2013, S. 77-78).

JournalistInnen bemühen sich bei ihrem Streben nach Objektivität um die Prüfung der Aussagen Dritter, während es WissenschaftlerInnen um die Prüfung eigener Annahmen geht (vgl. Post 2013, S. 130). Charakteristisch für das Objektivitätsverständnis von JournalistInnnen ist es, „Fakten für sich sprechen [zu] lassen“ und „Sachverhalte realitätsgerecht darzustellen“ (Post 2013, S. 138), während es bei WissenschaftlerInnen um Messbarkeit, Systematik und Nachvollziehbarkeit sowie die kritische Kontrolle eigener Vorstellungen geht (vgl. Post 2013, S. 138). Übertragen auf die professionelle Praxis, wird dieser Unterschied besonders bei der Kommunikation von Risiken deutlich: ,Journalisten stellen Risiken häufig als einzelne, (eingetretene oder potentielle) Stör- oder Schadensfälle dar, während Wissenschaftler Einzelfälle im Kontext der Häufigkeit ihres (tatsächlichen oder potentiellen) Auftretens erfassen." (Post 2013, S. 19-20) Dies führt unter anderem dazu, dass WissenschaftlerInnen häufig eine Dramatisierung von Forschungsergebnissen in den Medien beklagen (vgl. Post 2013, S. 20). Bei der Betrachtung von differenzierten Systemen ist es nicht weiter überraschend, dass die systemimmanenten Prozesse, Normen und Werte nicht störungsfrei miteinander korrespondieren.

Die Systeme Wissenschaft und Journalismus sind also abgrenzbar, und aus ihren unterschiedlichen Codes und Programmen resultieren auch unterschiedliche Praktiken. Im Hinblick auf unseren Forschungsgegenstand, die Bewertung von Klimajournalismus durch Wissenschaft, ergibt sich daher die Frage, ob und wie die Bewertungen journalistischer Beiträge die funktionalen Differenzen der Systeme Wissenschaft und Journalismus widerspiegeln. Die Forschungsfragen lauten:

Forschungsfrage 1 Welche Qualitätskriterien fordern die WissenschaftlerInnen im Journalismus ein?

Forschungsfrage 2 Wie bewerten sie auf dieser Grundlage die Leistungen des Klimajournalismus? 
Forschungsfrage 3 Inwieweit sind die geforderten Qualitätskriterien den Systemen Wissenschaft und Journalismus zuzuordnen?

\section{Klimajournalismus-Watchblog als Fallstudie}

Um die Forschungsfragen zu beantworten, wurde ein Klimajournalismus-Watchblog als Fallstudie ausgewählt. Die öffentliche Debatte über das Thema Klimawandel eignet sich für diese Analyse, da sie sowohl die Wissenschaft als auch den Journalismus vor besondere Herausforderungen stellt. Diese können unter dem Label ,,postnormal" zusammengefasst werden. Dies bedeutet, dass die Arbeit von WissenschaftlerInnen Fragen von großer gesellschaftlicher Bedeutung, von hoher Unsicherheit und von Wertkonflikten betrifft (vgl. Funtowicz und Ravetz 1993). Blogs, die sich thematisch mit dem Klimawandel auseinandersetzen, zählen zu den am weitesten verbreiteten Wissenschaftsblogs (vgl. Scheloske 2012, S. 268). Als Wissenschaftsblog gelten dabei Online-Angebote, die sich entweder dadurch auszeichnen, dass die AutorInnen aus dem Wissenschaftsbereich stammen, oder durch die Ausrichtung auf wissenschaftliche Themen (vgl. Scheloske 2012, S. 267-268). Eine weitere Blogform bilden Watchblogs, die als Plattform zur Äußerung von Kritik dienen. Medienkritische Weblogs befassen sich dabei fast ausschließlich mit Medienthemen und setzten sich kritisch mit deren Inhalten auseinander (vgl. Hutter 2009, S. 86). Kontinuität wird bei der Bereitstellung von solchen Inhalten vorausgesetzt (vgl. Hutter 2009, S. 86). Die Inhalte eines Medien-Watchblogs zeichnen sich durch Kritik beziehungsweise Beobachtung journalistischer Berichterstattung aus, wobei sie sich auf ein oder mehrere Medienangebote beziehen können und thematisch festgelegt oder offen sind (vgl. Wied und Schmidt 2008, S. 180). Sie können somit zur ,offenen Kritikkultur“ durch JournalistInnen beitragen (vgl. Wied und Schmidt 2008, S. 184).

\subsection{Fallauswahl und Stichprobe}

Die empirische Analyse dieser Studie beruht auf dem englischsprachigen Blog Climate Feedback, der in seiner Form einzigartig ist. Climate Feedback greift das Kritik-Prinzip des Peer-Reviews auf und wendet es auf journalistische Beiträge an, das heißt, wissenschaftseigene Verfahren werden auf einen außersystemischen Bereich angewandt. Der Blog nutzt beispielhaft die Errungenschaften des Web 2.0 und veröffentlicht wissenschaftliches Feedback über Klimaberichterstattung in englischsprachigen Medien. Über 180 WissenschaftlerInnen ${ }^{1}$ verschiedener Nationalitäten tragen zu den „Climate Feedbacks“ bei und evaluieren Berichte von englischsprachigen Nachrichtenmedien wie etwa Daily Mail, The Wall Street Journal, Forbes oder The Guardian. Auch in der Medienberichterstattung selbst wurde die Arbeit von Climate Feedback aufgegriffen (z. B. Deutsche Welle 2018; Independent 2017; The Guardian 2017; The Huffington Post 2017) und kann daher als potentieller FeedbackMechanismus wirken. Andere Medien-Watchblogs, wie Carbon Brief oder Medien-

\footnotetext{
1 Stand April 2019 (vgl. Climate Feedback o.J.a)
} 


\section{Analysis of "2014 Breaks Heat Record, Challenging Global Warming Skeptics"}

Published in The New York Times, by Justin Gillis on 16 Jan. 2015

Eight climate scientists have analyzed the article and they estimate its overall scientific credibility to be 'high' to 'very high'?

A majority of reviewers tagged the article as: Accurate, Insightful, Sound reasoning.

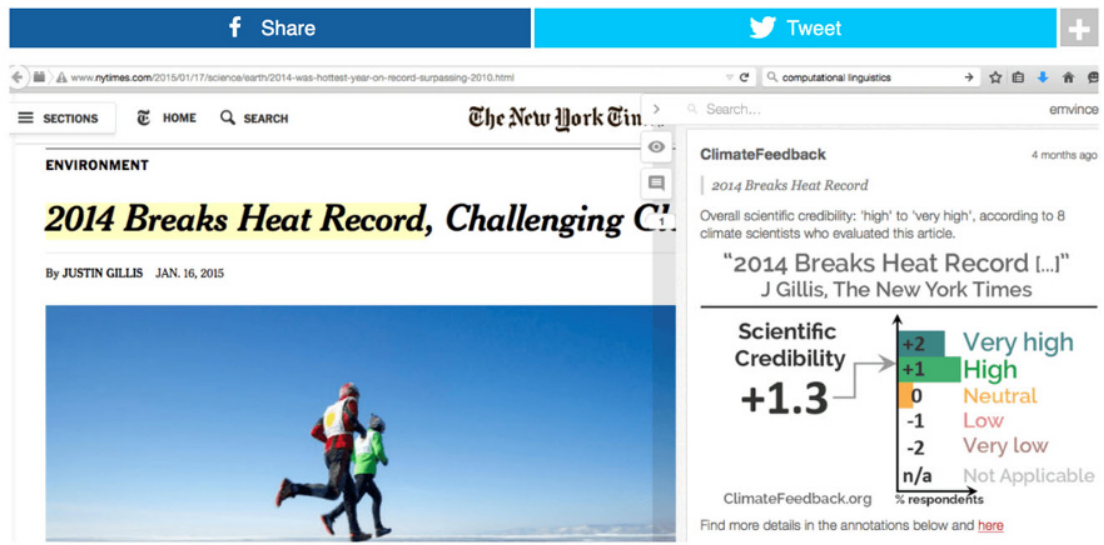

\section{SCIENTISTS' FEEDBACK}

\section{SUMMARY}

This article covers the release of annual average global surface temperature estimates for 2014, which placed it as the warmest year on record. Justin Gillis' article placed the 2014 temperature record in the context of both natural climate variability and the long-term trend of anthropogenic climate change. Gillis fairly described some ideas as still subject to debate in the scientific community, while identifying consensus ideas clearly.

See all the scientists' annotations in context

\section{REVIEWERS' OVERALL FEEDBACK}

These comments are the overall opinion of scientists on the article, they are substantiated by their knowledge in the field and by the content of the analysis in the annotations on the article.

\section{John Dwyer, Postdoctoral research fellow, MIT:}

This article accurately describes global warming and puts the news that 2014 is the hottest year on record into appropriate context. The article does a very good job of distinguishing between climate variability and climate change with helpful discussion on ENSO and the relatively cold temperatures in the Eastern United States (while staying within the bounds of the mainstream climate science understanding).

Abb. 1 Beispiel für die Darstellung eines wissenschaftlichen Feedbacks auf climatefeedback.org (Quelle: Climate Feedback 2015)

Doktor Umwelt, sind keine rein wissenschaftlichen Angebote, da sich hier sowohl WissenschaftlerInnen als auch JournalistInnen unter den AutorInnen finden. Sie sind daher nicht für diese Analyse geeignet. 
Das Ziel von Climate Feedback ist es, ,to help Internet users-from the general public to influential decision-makers-distinguish inaccurate climate change narratives from scientifically sound and trustworthy information in the media“ (Climate Feedback o.J.b). Finanziert wird das Projekt primär vom Center for Climate Communication der University of California, Merced, dem Center for Information Technology Research in the Interest of Society (CITRIS) sowie aus Spenden und über Crowdfunding (vgl. Climate Feedback o.J.c).

Artikel, die auf Climate Feedback kommentiert werden, enthalten für die Klimadebatte relevante Schlagwörter (z.B. „,climate change“, ,global warming“, ,ocean acidification“, „carbon dioxide“, „sea level rise“) und hatten eine hohe Resonanz auf Social-Media-Plattformen. Nach dem Sichten der Artikel entscheiden die Blogautoren Emmanuel Vincent und Scott Johnson, ob darin einerseits verifizierbare Aussagen enthalten sind und der journalistische Text andererseits den Anspruch auf wissenschaftliche Belastbarkeit erhebt. Die so ausgewählten Artikel werden dann WissenschaflterInnen, die Expertise in dem jeweiligen Bereich haben, zur Begutachtung vorgelegt. ${ }^{2}$

Auf climatefeedback.org sind die Ergebnisse dieser Gutachten unter der Rubrik „Article Reviews“ zu finden. Dort ist zu jedem analysierten journalistischen Beitrag eine Zusammenfassung der Feedbacks in Form eines Blogbeitrags vorhanden (s. Abb. 1). Die Anmerkungen lassen sich im Detail auch direkt am journalistischen Beitrag nachvollziehen.

Die Stichprobe dieser Studie umfasst alle „Article Reviews“ vom 29.01.2015, dem Tag des ersten veröffentlichten Reviews, bis zum Abschluss der Untersuchung am 04.12.2017, die einen journalistischen Beitrag über den Klimawandel bewerteten $(N=82){ }^{3}$ Die Stichprobe zeigt eine diverse Zusammensetzung: Insgesamt liegen den Bewertungen journalistische Beiträge aus 28 verschiedenen Medien zugrunde (s. Tab. 3 im Anhang).

\subsection{Methode}

Da es sich um eine explorative Studie handelt, die die Interpretation von Texten erfordert, ist eine qualitative Inhaltsanalyse angemessen (vgl. Schreier 2012, S. 21). Sie eignet sich, um latente Sinnstrukturen von Inhalten herauszuarbeiten (vgl. Schreier 2012, S. 2). Wir folgen dabei dem Ansatz von Schreier (2012), der trotz der qualitativen Analyse und des induktiven Vorgehens eine Quantifizierung von Codierhäufigkeiten erlaubt und auch die Überprüfung der Reliabilität der Codierung empfiehlt. Ausgehend von den Objekten der Kritik haben wir uns dabei zu den Kriterien der Kritik hochgearbeitet, die wir durch die Interpretation wiederkehrender Objekte identifiziert haben. Dies erlaubt es uns, die abstrakten Kriterien in der wissenschaftlichen Kritik zu identifizieren.

\footnotetext{
2 Die Informationen über das Vorgehen stammt aus einer persönlichen Email-Korrespondenz mit Blogautor Emmanuel Vincent.

3 Nur ein Blogeintrag vom 22.06.2015 wurde von der Analyse ausgeschlossen, da er keinen journalistischen Beitrag, sondern eine päpstliche Enzyklika analysiert.
} 


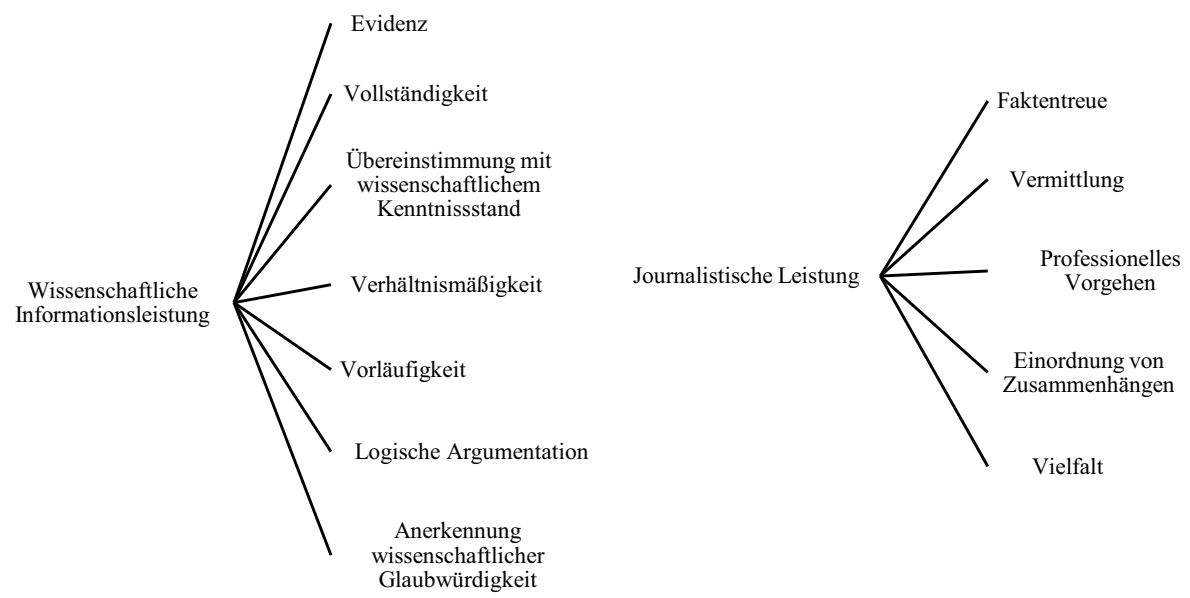

Abb. 2 Aufbau des Kategoriensystems

Die qualitative Inhaltsanalyse wurde mit Hilfe der Software MAXQDA durchgeführt. Das Kategoriensystem haben wir induktiv an den Texten entwickelt. Im zweiten Schritt diente das Kategoriensystem als Grundlage für die systematische und auch quantifizierende Beschreibung des Untersuchungsmaterials. Das Kategoriensystem besteht aus zwei übergeordneten Dimensionen und zwölf Kategorien (s. Abb. 2). Bei den Kategorien wurde zudem erfasst, ob sie positiv oder negativ Erwähnung fanden, ob also ihr Vorhandensein gelobt oder ihr Fehlen kritisiert wurde. Die Dimensionen stellen die Zuordnung zum System Wissenschaft bzw. Journalismus dar, und die Kategorien beschreiben die identifizierten Qualitätskriterien. ${ }^{4}$ Die Reliabilität der Codierung auf Grundlage des Kategoriensystems wurde durch einen Reliabilitätstest anhand einer zufällig ausgewählten Stichprobe überprüft. Hierzu wertete eine zweite Codiererin die ausgewählten „Article Reviews“ $(N=10)$ mit dem Kategoriensystem inhaltsanalytisch aus. Die Übereinstimmung der Codiereinheiten $(N=448)$ wurde anhand eines Reliabilitätstests ermittelt. Der Test ergab einen Kappa Koeffizienten von 0,67 für die Dimension ,wissenschaftliche Informationsleistung“ und 0,69 für die Dimension ,,journalistische Leistung“. Der im Vergleich zu quantitativen Inhaltsanalysen relativ niedrige Kappa Koeffizient kann angesichts der qualitativen Charakteristik der Untersuchung und dem komplexen Kategoriensystem als Indikator hinreichender Reliabilität gelten (vgl. Landis und Koch 1978).

\section{Ergebnisse}

Um besser zu verstehen, nach welchen Kriterien WissenschaftlerInnen journalistische Beiträge über den Klimawandel bewerten, betrachten wir zunächst, von wem

\footnotetext{
${ }^{4}$ Neben den Kategorien wurden auch formale Variablen mit Bezug zum Blogpost (z. B. das Veröffentlichungsdatum und der Name der AutorInnen) und zum zugrunde liegenden journalistischen Beitrag (z.B. Überschrift und Darstellungsform). Die Studie wurde nicht durch externe Mittel finanziert.
} 
die Feedbacks kommen, die ausgewertet wurden. Die Bewertung eines Artikels fundiert auf der Synthese mehrerer Reviews: Die Spanne reicht von drei bis siebzehn WissenschaftlerInnen, die an dem Review eines Textes beteiligt sind. Durchschnittlich bewerten sieben wissenschaftliche KommentatorInnen einen journalistischen Beitrag. Pro Artikel fasst Climate Feedback mithilfe der Kommentare der GutachterInnen die allgemeine wissenschaftliche Glaubwürdigkeit auf einer Skala von 2 (sehr hoch) bis -2 (sehr gering) zusammen. Lediglich ein Bloomberg-Artikel erhält dabei die Bestnote, wohingegen gleich 6 Artikeln aus The Australian, New York Post, The Boston Globe und The Spectator eine sehr geringe Glaubwürdigkeit attestiert wird (s. Tab. 3 im Anhang). Durchschnittlich werden die Artikel mit einer leicht negativen Tendenz bewertet $(-0,24)$. Doch was genau sind dabei die Kritikpunkte der WissenschaftlerInnen?

Um diese Frage zu beantworten, wurden insgesamt 82 Blogbeiträge mit den Kommentaren von 184 WissenschaftlerInnen ausgewertet. Die Hälfte der WissenschaftlerInnen $(N=96)$ taucht nur einmal als ReviewerIn auf (Abb. 3 im Anhang). Nur die Kommentare von fünf WissenschaftlerInnen sind deutlich häufiger (mehr als zehnmal) in der Stichprobe vertreten. Das zeigt, dass die wissenschaftlichen Gutachten die Anforderungen vieler verschiedener Personen aus der Wissenschaft abbilden und nicht nur die Kriterien weniger Menschen dominieren.

Wie bereits erwähnt, wurden Kategorien unterschieden, die sich auf die wissenschaftliche Informationsleistung beziehen, und solche, die sich auf die journalistische Leistung beziehen. Die Dimension „,wissenschaftliche Informationsleistung“ betrifft Bewertungen, die sich auf die Wissenschaftlichkeit der Berichterstattung beziehen: Ist die Darstellung wissenschaftlich belastbar? Die Kategorien zeichnen sich also dadurch aus, dass Maßstäbe des Systems Wissenschaft an den journalistischen Beitrag angelegt wurden. Die Dimension ,,journalistische Leistung“ erfasst hingegen Bewertungen des journalistischen Produkts: Wie ist die journalistische Umsetzung gelungen? Mit 1150 Codierungen fallen die meisten Bewertungen unter die Dimension ,,journalistische Leistung“. Bewertungen, die der Dimension ,,wissenschaftliche Informationsleistung“ zugeordnet wurden, belaufen sich auf insgesamt 778 codierte Einheiten.

\subsection{Kriterien zur Beurteilung der wissenschaftlichen Belastbarkeit journalistischer Berichterstattung}

Es wurden sieben Kategorien (s. Tab. 1) entwickelt, die sich der Dimension ,wissenschaftliche Informationsleistung" zuordnen lassen und erfassen, anhand welcher Kriterien WissenschaftlerInnen die wissenschaftliche Belastbarkeit eines Textes festmachen: Neben den aufgeführten Belegen (,Evidenz“) ist die „Übereinstimmung mit dem wissenschaftlichen Kenntnisstand“ sowie die „Vollständigkeit“ der Darstellung in die Bewertung der wissenschaftlichen Informationsleistung eingeflossen. Die WissenschaftlerInnen fordern außerdem ,Verhältnismäßigkeit“ bei der Wiedergabe, die Beachtung von Limitierungen und Unsicherheiten (,Vorläufigkeit“) und eine ,logische Argumentation“ sowie ganz grundsätzlich die „Anerkennung wissenschaftlicher Glaubwürdigkeit“. Die Kategorien werden im Folgenden anhand von Beispielen genauer erläutert. 
Tab. 1 Verteilung der Kategorien innerhalb der Dimension wissenschaftliche Informationsleistung

\begin{tabular}{llll}
\hline Kategorie & $N$ gesamt & $\begin{array}{l}N \text { positive } \\
\text { Bewertung }\end{array}$ & $\begin{array}{l}N \text { negative } \\
\text { Bewertung }\end{array}$ \\
\hline Evidenz & 217 & 62 & 155 \\
Vollständigkeit & 158 & 17 & 141 \\
Übereinstimmung wiss. Kenntnisstand & 164 & 38 & 126 \\
Verhältnismäßigkeit & 88 & 7 & 81 \\
Vorläufigkeit & 60 & 26 & 34 \\
Logische Argumentation & 39 & 2 & 37 \\
Anerkennung wiss. Glaubwürdigkeit & 52 & 0 & 52 \\
Gesamt & 778 & 152 & 626 \\
\hline
\end{tabular}

Die Kategorie „Evidenz“ kommt am häufigsten vor $(N=217)$, was zeigt, dass Urteile zur wissenschaftlichen Belastbarkeit eines journalistischen Beitrags wesentlich durch die verwendeten Belege gekennzeichnet sind. In 62 Fällen wurden die verwendeten Belege zu Aussagen positiv erwähnt, mehr als doppelt so oft $(N=155)$ wurde allerdings Kritik geäußert. Die Kommentare gaben neben allgemeinen Aussagen über die Fundierung eines Beitrags auch Aufschluss darüber, was unter legitimen wissenschaftlichen Evidenzen überhaupt verstanden wird. So ist WissenschaftlerInnen etwa die Wahl von zuverlässigen Quellen wichtig.

Meinungsäußerungen werden in keinem Fall positiv erwähnt, sondern als Abwesenheit einer zulässigen Argumentationsgrundlage betrachtet. Die meinungsbetonte Diskussion wissenschaftlicher Fragen wird als unzulässig betrachtet: "The article speaks about scientific questions under an 'opinion' banner-as if questions about the role of $\mathrm{CO}_{2}$ in the Earth system could be a matter of opinions."

Als gültige Belege gelten hingegen Studien, die ein Peer-Review-Verfahren durchlaufen haben, oder Aussagen, die von qualifizierten ExpertInnen stammen. Wissenschaftliche Reputation ist dabei der Maßstab für Qualifikation. Daneben finden die institutionelle Herkunft und die Expertise der Person im jeweiligen Feld als Kriterien Erwähnung. Expertise wird eindeutig Personen aus der Wissenschaft zugesprochen, wie auch in folgendem Zitat deutlich wird: „As it is more an organized survey of scientists rather than a non-science author trying to make a scientific argument."

Neben wissenschaftlichen Belegen ist auch wichtig, dass der aufgeführte Beleg in direktem Zusammenhang mit der zu belegenden These steht, was in der folgenden Textstelle deutlich wird: „The article misuses a Nature article on a geological process 90 millions [sic!] years ago to argue the warming of the past century is not anthropogenic.“ Ein journalistischer Beitrag kann jedoch trotz Abzügen bei der Eignung eines Belegs noch eine gute Bewertung erhalten.

Die „Übereinstimmung mit wissenschaftlichem Kenntnisstand“ ( $N=164)$, also die Wiedergabe von Informationen entsprechend dem Stand der Wissenschaft, ist ebenfalls eine zentrale Anforderung der Wissenschaft an den Journalismus. Kritik $(N=126)$ und Lob $(N=38)$ innerhalb dieser Kategorie sind ungleich verteilt: Es wird also mehr kritisiert, dass Darstellungen von Stand der Forschung abweichen, als dass gelobt wird, sie repräsentierten diesen korrekt. Aktualität, bezogen auf den Forschungstand, ist ebenso wie die Konsistenz mit Forschungsergebnissen ein 
positives Merkmal: Je häufiger eine wissenschaftliche These in Studien bestätigt wurde, als desto belastbarer gilt sie.

Die Missachtung breiter wissenschaftlicher Einigkeit über einen Sachverhalt und die Platzierung von AußenseiterInnen-Standpunkten, gelten dementsprechend als negativ. Zumindest sollten diese deutlich als solche gekennzeichnet werden: „The article contains numerous statements that are not supported by the scientific literature, but instead appear to be the personal opinion of Professor Wadhams. It is difficult to provide any meaningful rating for such an interview piece-Wadhams has the right to share his opinions when asked (however unsupported they may be) and at times even makes it clear that his statements are far outside the scientific mainstream."

Noch mehr Kritik $(N=126)$ äußerten die KommentatorInnen an der unvollständigen Wiedergabe wissenschaftlicher Informationen. Die Kategorie „Vollständigkeit““ wurde insgesamt 158-mal codiert, davon nur 17-mal in positiver Ausprägung. Negativ zur Sprache kommt an vielen Stellen entweder die Vernachlässigung relevanter Aspekte oder die selektive Auswahl von Aspekten, sogenanntes „Cherry-Picking“. Die selektive Abbildung von Fakten stellt eine eindeutige Unzulänglichkeit dar. Enthält ein Beitrag dagegen Zusatzinformationen, wurde dies positiv hervorgehoben, wobei dies eher als Optimierung und nicht eine notwendige Grundvoraussetzung betrachtet wird.

Die wissenschaftliche Belastbarkeit zeigte sich nicht nur in der Konformität mit wissenschaftlichen Ergebnissen, sondern auch an der ,,Verhältnismäßigkeit“ der Darstellung. Hier liegen insgesamt nur 7 von 88 Codierungen vor, die eine positive Ausrichtung besitzen. In diesen haben die WissenschaftlerInnen überwiegend die Abwesenheit von übertriebenen Darstellungen gelobt: „,This article was well substantiated and measured in its reporting (...) without being hysterical about the potential for mass fish suffocations, which I have seen in previous articles on this subject“". Kritik wird laut an übertriebenen Darstellungen, Bagatellisierung sowie zurückhaltenden Darstellungen. In einigen Fällen bezieht sich die Kritik auf Überschriften: ,The title is possibly a little overstated-I might instead say that the AMOC [Atlantic Meridional Overturning Circulation, Anmerkung d. AutorInnen] is at risk of collapsing in a warming world." Andere Äußerungen beziehen sich auf die mögliche Wirkung auf die Lesenden: „Still the reader may get the impression that the changes in precipitation are larger than they most likely are." Kritik wurde außerdem geäußert, wenn wissenschaftliche Erkenntnisse als bahnbrechend dargestellt wurde, ohne es zu sein.

Auch die Forderung nach Beachtung der „Vorläufigkeit“ von Befunden $(N=60)$ kommt wiederholt zur Sprache. Häufig geht es um die Frage, ob Unsicherheiten oder Grenzen wissenschaftlicher Aussagen beachtet werden. Lob $(N=26)$ und Kritik $(N=34)$ sind dabei vergleichsweise ausgewogen. Es zeigt sich, dass die Erwähnung von Vorläufigkeit als Merkmal eines guten Beitrags gilt. Häufig bezieht sich die Kritik auf unzulässige Verknüpfungen von Wetter- mit Klima-Phänomenen und damit verbundene wissenschaftlichen Unsicherheiten: „There remains real uncertainty about the link between Arctic warming and certain mid-latitude weather patterns, which the article does not make entirely clear to the reader.“ Ein Hauptaugenmerk 
der Kritik lag außerdem auf der Berücksichtigung von ungeklärten Aspekten wissenschaftlicher Fragestellungen.

Kritisch sehen die GutachterInnen auch fehlende Stringenz in der Argumentation. Unter der Kategorie „logische Argumentation“ $(N=39)$ wird in fast allen Fällen $(N=37)$ deren Fehlen beklagt. Neben mangelhafter Stringenz in der Argumentation bezieht sich eine wiederkehrende Kritik auf die Verwendung von StrohmannArgumenten.

Ganz grundsätzlich fordern die WissenschaftlerInnen die „Anerkennung wissenschaftlicher Glaubwürdigkeit“ $(N=52)$. Die Kategorie kam ebenfalls fast ausschließlich als Klage über das Fehlen dieser Anerkennung vor. Kritik wird etwa an der Ablehnung von Wissenschaft oder an klimaleugnerischen Argumentationen geübt: „This opinion in The Australian by Ian Plimer repeats a number of false but common claims to support an argument that human activities are not responsible for climate change, and that renewables like wind and solar cannot effectively replace fossilfuel-burning power plants." Auch grundlegende Skepsis gegenüber Forschungsmethoden oder dem Wissenstand sowie Beschwerden über diffamierende Aussagen kommen zur Sprache: “The article also rallies emotively against 'Global Quackery' from thousands of qualified climate scientists of all political persuasions."

\subsection{Kriterien zur Beurteilung der journalistischen Leistung}

Die zweite Dimension der Kriterien, die WissenschaftlerInnen zur Beurteilung journalistischer Artikel heranziehen, ist die ,,journalistische Leistung“. Um diese Dimension zu erfassen, wurden fünf Kategorien entwickelt (s. Tab. 2). Es handelt sich um Kommentare zur journalistischen Umsetzung, die zu einem großen Teil der Vermittlungsleistung des Beitrags gelten (,Vermittlung“). Als zentrales Gütekriterium von WissenschaftlerInnen stellt sich außerdem die Einhaltung der Sorgfaltspflicht heraus: Der Wahrheitsanspruch (,Faktentreue“) sowie die Einhaltung professioneller journalistischer Standards (,professionelles Vorgehen“) werden hier angebracht. Ein weiteres Augenmerk liegt auf dem Kontext (,Einordnung von Zusammenhängen“), in den die Informationen eingebettet werden, sowie die Berücksichtigung verschiedener Positionen und Quellen (,Vielfalt“).

Innerhalb der Kommentare, die sich auf die journalistische Leistung beziehen, kommt die „Vermittlung“ am häufigsten zur Sprache $(N=351)$. Unter der Kategorie wurden Aussagen erfasst, die eine Bewertung der Vermittlungsleistung des Beitrags

Tab. 2 Verteilung der Kategorien innerhalb der Dimension journalistische Leistung

\begin{tabular}{llll}
\hline Kategorie & $N$ gesamt & $\begin{array}{l}\text { N positive } \\
\text { Bewertung }\end{array}$ & $\begin{array}{l}N \text { negative } \\
\text { Bewertung }\end{array}$ \\
\hline Vermittlung & 351 & 161 & 190 \\
Faktentreue & 331 & 114 & 217 \\
Professionelles Vorgehen & 217 & 96 & 121 \\
Einordnung von Zusammenhängen & 177 & 84 & 93 \\
Vielfalt & 74 & 42 & 32 \\
Gesamt & 1150 & 497 & 653 \\
\hline
\end{tabular}


enthalten. Im Vergleich zur wissenschaftlichen Informationsleistung sind Bewertungen der Vermittlungsfunktion des Journalismus ausgeglichener, auch wenn negative Kommentare $(N=190)$ leicht gegenüber positiven $(N=161)$ überwiegen. Ein Großteil der Kritik bezieht sich auf irreführende Darstellungen: „This piece misleads its readers into thinking positive impacts of climate change could outweigh the negative impacts." Ein weiterer Aspekt ist der Informationsgehalt von Beiträgen. Die WissenschaftlerInnen erwarten von journalistischen Beiträgen, dass sie Lernprozesse anstoßen. Zudem kritisieren und loben sie auch die Wahl bestimmter Formulierungen: Hier stellt Genauigkeit ein wiederkehrendes Gütesiegel dar. Und zuletzt ist Lob an der gelungenen Vereinfachung wissenschaftlichen Wissens zentral: „The article does a very good job in working out the main message of the original scientific paper in an understandable way without oversimplification.“

Auch die „Faktentreue“ $(N=331)$ wird häufig evaluiert. Hier überwiegt ebenfalls die Kritik $(N=217)$. Genauigkeit in der Darstellung wird gelobt. Abweichungen oder selbstständige Ergänzungen der Originalinformation werden kritisiert, ebenso wie inhaltlich falsch zitierte Studien und ExpertInnen. Letztere kommen in einigen Climate Feedbacks selbst zu Wort, wie etwa Howard Browman, der sich in einem Beitrag der Times nicht wahrheitsgemäß wiedergegeben sieht: „The Times article is not representative of the message that I tried to give the journalist during the interview - that is, the message presented my introduction to the special issue in which I state clearly and explicitly that ocean acidification IS happening and WILL have effects."

Unter der Kategorie ,professionelles Vorgehen“, insgesamt 217 Mal codiert, kommen 96 positive und 121 negative Bewertungen vor. Die Kommentare beziehen sich auf die Unparteilichkeit des Beitrags, bewerten die Recherche und auch die Themenwahl. Eine geeignete Themenwahl zeichnet sich aus Sicht der WissenschaftlerInnen durch Aktualität, Neuigkeit und Relevanz aus. Letzteres tritt in Kommentaren hervor, welche die Wichtigkeit von wissenschaftlichen Themen herausstellen: „We certainly need to increase people's understanding of this problem." Kritik an der journalistischen Recherche äußerte sich überwiegend in der als mangelhaft empfundenen Überprüfung von Fakten oder Aussagen von zitierten Personen. Gleichzeitig finden sich wiederholt Beurteilungen über die wissenschaftliche Kompetenz der JournalistInnen. Grundlegende Zweifel an deren wissenschaftlichen Verständnisses führen ebenso zur Aberkennung von Kompetenz wie fehlende Fachkenntnis. Es kam auch durchaus vor, dass die Glaubwürdigkeit des Mediums aufgrund des bewerteten Artikels grundsätzlich in Frage gestellt wird: „People should know that Forbes is nowadays just a blogging platform."

Nahezu ausgeglichen war Lob $(N=84)$ und Kritik $(N=93)$ hinsichtlich der Aufgabe von JournalistInnen, Informationen so einzubetten, dass LeserInnen sie verstehen, was unter „Einordnung von Zusammenhängen“ $(N=177)$ erfasst wurde. Für die WissenschaftlerInnen ist zum einen die Kontextualisierung ein entscheidender Faktor, wobei oftmals die Einbettung in den größeren Zusammenhang positiv betont wird. Aber auch Informationen vor den Hintergrund aktueller Forschung zu stellen, wird als wichtige Orientierungsleistung betrachtet: „I think it could have provided more context on what is known (or not known) about current, observed changes in floods." Daneben ist zentral, dass die Verständlichkeit der Information nicht beein- 
trächtigt ist: ,Some others lack the necessary context to be clearly understood by the reader." Positiv wird hervorgehoben, wenn JournalistInnen die Relevanz der jeweiligen Informationen erklären. Es gilt außerdem der Anspruch, zwischen kurzfristigen Klimaschwankungen und langfristigen Klimaveränderungen zu unterscheiden. Beim Umgang mit Klimadaten sollen JournalistInnen deren Aussagekraft einordnen, zum Beispiel erklären, auf welche Raum- und Zeitdimension sie anwendbar sind: „Care should be taken not to misinterpret short-term variations in local sea level rise trends, such as the higher rate of sea level rise in Florida over the past decade, which is not expected to continue over the long term."

Am seltensten ziehen WissenschaftlerInnen das Kriterium „Vielfalt“ $(N=74)$ zur Beurteilung journalistische Leistungen heran. Wieder treten Lob $(N=32)$ und Kritik $(N=42)$ etwa gleichermaßen auf. Gegenstand von Kritik ist das Auslassen von kritischen Gegenüberstellungen: ,The author of this article did not interview anyone that Dr Browman is criticizing." Beanstandet werden auch Versäumnisse, verschiedene Seiten einer Debatte abzubilden: ,There are skeptical voices in the community whose views differ from those presented in this article. A more complete story could have included them to provide an alternative perspective on the role of a changing Arctic." Dementsprechend positiv empfunden wird die Vermittlung auch von Meinungsverschiedenheiten: „One thing I like is that it mentions different opinions on some points where scientists do not agree rather than giving a biased story.“

\section{Zusammenfassung, Diskussion und Ausblick}

Die zentrale Frage dieser Studie war, welche Qualitätskriterien die Wissenschaft an den (Wissenschafts-)Journalismus anlegt, welche Aspekte sie also lobt und kritisiert. Zur Beantwortung haben wir eine qualitative Inhaltsanalyse des Medien-Watchblogs Climate Feedback durchgeführt, auf dem WissenschaftlerInnen journalistische Beiträge über den Klimawandel bewerten. Die jeweils kritisierten Einzelaspekte wurden unter eine Reihe von Qualitätskriterien subsummiert. Diese Kategorien ließen sich ihrerseits plausibel den Systemen Journalismus und Wissenschaft unter den Dimensionen ,wissenschaftliche Informationsleistung“ und ,,journalistische Leistung“ zuordnen. Dass dies möglich war, bestätigt zunächst einmal, dass die Systemtheorie als Heuristik für empirische Analysen dieser Art durchaus anwendbar ist - trotz Uneinigkeiten unter den Systemtheoretikern, wie der Leitcode des Journalismus genau zu benennen ist und trotz der Überlappungen im Leitbild von Wissenschaft und Journalismus und Tendenzen zu Grenzverwischungen, die wir im Theorieteil skizziert haben.

Unsere Ergebnisse zeigen, dass sich WissenschaftlerInnen in ihrer Kritik überraschenderweise häufiger auf die journalistische Leistung $(N=1150)$ als auf die wissenschaftliche Informationsleistung $(N=778)$ beziehen. Sie beschäftigen sich also keineswegs überwiegend mit der wissenschaftlichen Performance der JournalistInnen, sondern messen sie an journalistischen Kriterien. Diese bewerten sie deutlich positiver: Während die journalistische Leistung von Wissenschaftsjournalismus in $43 \%$ der Fälle positiv bewertet werden, sind es nur $20 \%$ bei der wissenschaftlichen Informationsleistung. 
Hinsichtlich der wissenschaftlichen Informationsleistung zeigen die Ergebnisse dieser Studie, dass sich die Qualitätskriterien von WissenschaftlerInnen stark an Kriterien orientieren, die auch für ihre eigene Arbeit relevant sind: Evidenz, Vollständigkeit, die Übereinstimmung mit dem wissenschaftlichen Kenntnisstand, die Verhältnismäßigkeit und Vorläufigkeit von wissenschaftlichen Ergebnissen sowie eine logische Argumentation. Dass auch die Anerkennung von wissenschaftlicher Glaubwürdigkeit als Kriterium von Bedeutung ist, zeigt, dass WissenschaftlerInnen in Zeiten von „fake-science“ eine pauschale Skepsis gegenüber wissenschaftlichen Methoden und Ergebnissen kritisieren. Sie erwarten einen Vertrauensvorschuss für die Wissenschaft. Besonders die ersten drei Kriterien finden häufige Erwähnung, und ihr Fehlen wird kritisch angemerkt. Dies muss im Zusammenhang mit dem Schwerpunkt Klimawandel gesehen werden: Das weit erforschte wissenschaftliche Thema als politische Glaubensfrage zu diskutieren, wird als eine Methode der KlimaleugnerInnen angesehen (vgl. McKnight 2010, S. 703-704).

Die journalistische Leistung bewerten WissenschaftlerInnen an Hand von fünf Kriterien: der journalistischen Vermittlungsleistung, der Faktentreue eines Artikels, dem professionellen Vorgehen des/der JournalistIn, der Einordnung von Zusammenhängen sowie der Vielfalt an Positionen und Quellen. WissenschaftlerInnen bewerten hier ganz eindeutig Kernkompetenzen des Journalismus, die von journalistischen Normen und Werten bestimmt werden. Damit überschreiten WissenschaftlerInnen die eigenen Systemgrenzen und wenden nicht nur die Kriterien des eigenen Systems, sondern häufiger sogar die des Systems Journalismus an, um die Leistung von JournalistInnen zu beurteilen.

Das Blog Climate Feedback erweist sich somit als eine hybride Organisation an der Schnittstelle zwischen zwei Systemen. Es will mit einem am wissenschaftlichen Peer-Review angelehntem Verfahren die Qualität von Medienbeiträgen bewerten. Um Qualität zu steigern, wird eine systeminterne, qualitätssichernde Maßnahme auf ein außerwissenschaftliches System angewendet. Ebenso nennenswert ist die Ausrichtung der Blogbeiträge an Aktualität als journalistischem Kernprinzip. Die Erscheinungsdaten des journalistischen Beitrags und der Feedbacks zeigten, dass der überwiegende Teil der Bewertungen innerhalb einer Woche vollzogen wurde.

Obwohl die Systemtheorie berücksichtigt, dass JournalistInnen in der Lage sein müssen, die Relevanz von Ereignissen aus Sicht verschiedener Systeme zu identifizieren (vgl. Kohring 2016) und dass einzelne Akteure durchaus zwischen Systemen je nach Kontext wechseln können, so ergibt sich hier doch nicht nur ein kompetenter Wechsel von Individuen von einem System ins andere, sondern eine Gleichzeitigkeit von verschiedenen Systemlogiken, die schon in der Institution des Blogs angelegt ist. Dies ist auch eine Herausforderung für zukünftige Theoriebildung, die das Handeln von Akteuren und Systemlogiken noch stärker zusammendenkt, wie es in integrativen Ansätzen, zum Beispiel bei Schimank (2002, 2007), auch schon angelegt ist.

Ganz im Gegensatz zu Annahmen von Entdifferenzierung oder Entgrenzung beobachten wir die Gleichzeitig von eingeforderten Qualitätskriterien, die sich zwei verschiedenen Systemen zuordnen lassen, aber ohne dass darum die Grenzen verschwimmen. Vielmehr werden sie kompetent überschritten.

Damit liefert die Studie auch für den Medialisierungsansatz Impulse: Die Existenz des Blogs lässt sich als Medialisierungsbeleg anführen. Die explizite Zielsetzung der 
Online-Plattform, einflussreiche Medienbeiträge über den Klimawandel zu bewerten, ist ein Indikator dafür, dass Medienberichterstattung als relevant für die Erfüllung systemeigener Ziele der Wissenschaft erachtet wird beziehungsweise mit diesen in Konflikt geraten können. Es handelt sich bei den analysierten Kommentaren aber nicht um eine einseitige Übernahme von Medienlogiken, die die Wissenschaftslogiken dominieren. Wir finden Anzeichen eines kompetenten Umgangs von WissenschaftlerInnen mit journalistischen Beiträgen. Damit komplementieren die Medienkriterien die des eigenen Systems, ohne dass die Kriterien der Wissenschaftlichkeit fallengelassen oder ausgehöhlt werden. Bei den wissenschaftlichen Ansprüchen machen die Reviews keine Abstriche. Die Diffusion von Medienlogiken muss somit nicht zur Aufgabe von Logiken der Wissenschaft führen. Kompetente Akteure an der Schnittstelle zwischen Journalismus und Wissenschaft können Mehrsystemkompetenz erwerben und anwenden. Gerade das kann als Medialisierungsbeleg interpretiert werden, wenn man das Medialisierungskonzept entsprechend differenziert fasst und nicht als reine Dominanz von Medienlogiken in anderen Bereichen.

Eine Einschränkung dieser Studie ist die empirische Analyse eines einzelnen Blogs, der englischsprachige journalistische Artikel zum Thema Klimawandel kommentiert. Ein Vergleich von Climate Feedback mit anderen Plattformen ist jedoch aufgrund der einzigartigen Charakteristika nicht möglich, da es sich um ein neues Format der Beziehung von Wissenschaft und Journalismus handelt. Dennoch beinhaltet diese Studie das Feedback von WissenschaftlerInnen aus diversen Ländern. Durch die Inhaltsanalyse ihrer Kommentare war es möglich, wiederkehrende Argumente zu erkennen und Muster zu identifizieren, die sich möglicherweise auch außerhalb dieses Blogs wiederfinden lassen.

Da sich diese Studie mit der Bewertung der Medienberichterstattung über Klimawandel befasst, reflektieren die Ergebnisse vor allem die Meinungen von NaturwissenschaftlerInnen. Im Vergleich hierzu sind z. B. Interaktionen zwischen Medien und SozialwissenschaftlerInnen noch weniger gut erforscht (vgl. Fähnrich und Lüthje 2017) und bieten Anknüpfungspunkte für zukünftige Studien. Das Thema Klimawandel hat unserer Studie eine besondere Relevanz verliehen. Dennoch ist es auch ein polarisiertes, politisiertes, post-normales Thema. Es bleibt zu prüfen, welche Kriterien WissenschaftlerInnen an Wissenschaftsjournalismus zu anderen Themen anlegen. Wir vermuten, dass gerade die Besonderheiten der Klimadebatte die Ausbildung von Mehrsystemkompetenz auf Seiten der KlimawissenschaftlerInnen gefördert hat.

Funding Open Access funding enabled and organized by Projekt DEAL.

Open Access Dieser Artikel wird unter der Creative Commons Namensnennung 4.0 International Lizenz veröffentlicht, welche die Nutzung, Vervielfältigung, Bearbeitung, Verbreitung und Wiedergabe in jeglichem Medium und Format erlaubt, sofern Sie den/die ursprünglichen Autor(en) und die Quelle ordnungsgemäß nennen, einen Link zur Creative Commons Lizenz beifügen und angeben, ob Änderungen vorgenommen wurden.

Die in diesem Artikel enthaltenen Bilder und sonstiges Drittmaterial unterliegen ebenfalls der genannten Creative Commons Lizenz, sofern sich aus der Abbildungslegende nichts anderes ergibt. Sofern das betreffende Material nicht unter der genannten Creative Commons Lizenz steht und die betreffende Handlung nicht nach gesetzlichen Vorschriften erlaubt ist, ist für die oben aufgeführten Weiterverwendungen des Materials die Einwilligung des jeweiligen Rechteinhabers einzuholen. 
Weitere Details zur Lizenz entnehmen Sie bitte der Lizenzinformation auf http://creativecommons.org/ licenses/by/4.0/deed.de.

\section{Anhang}

Tab. 3 Zusammensetzung der Stichprobe nach Medium

\begin{tabular}{|c|c|c|}
\hline Medium & Anzahl Beiträge & Durchschnittliche Bewertung \\
\hline The New York Times & 10 & 1,46 \\
\hline The Guardian & 8 & 0,60 \\
\hline Forbes & 7 & $-1,04$ \\
\hline The Wall Street Journal & 7 & $-1,56$ \\
\hline The Washington Post & 7 & 1,54 \\
\hline Mashable & 5 & 1,32 \\
\hline The Telegraph & 4 & $-1,75$ \\
\hline Breitbart & 3 & $-1,93$ \\
\hline $\mathrm{CNN}$ & 3 & 0,30 \\
\hline The Atlantic & 3 & 1,60 \\
\hline The Daily Wire & 3 & $-1,73$ \\
\hline $\mathrm{BBC}$ & 2 & 1,20 \\
\hline The Australian & 2 & $-2,00$ \\
\hline The Independent & 2 & $-1,05$ \\
\hline Think Progress & 2 & 0,65 \\
\hline USA Today & 2 & 1,50 \\
\hline Bloomberg & 1 & 2,00 \\
\hline Daily Mail & 1 & $-1,90$ \\
\hline Grist & 1 & 0,80 \\
\hline Los Angeles Times & 1 & 0,80 \\
\hline New York Magazine & 1 & $-0,70$ \\
\hline New York Post & 1 & $-2,00$ \\
\hline Rolling Stone & 1 & $1 /-1$ \\
\hline The Boston Globe & 1 & $-2,00$ \\
\hline The Daily Caller & 1 & $-0,60$ \\
\hline The Spectator & 1 & $-2,00$ \\
\hline The Washington Times & 1 & $-1,90$ \\
\hline Vox & 1 & 1,70 \\
\hline Gesamt & 82 & $-0,24$ \\
\hline
\end{tabular}

Die Werte zur durchschnittlichen Bewertung stammen von den Climate-Feedback-Artikeln und wurden von den AutorInnen pro Medium zusammengefasst 


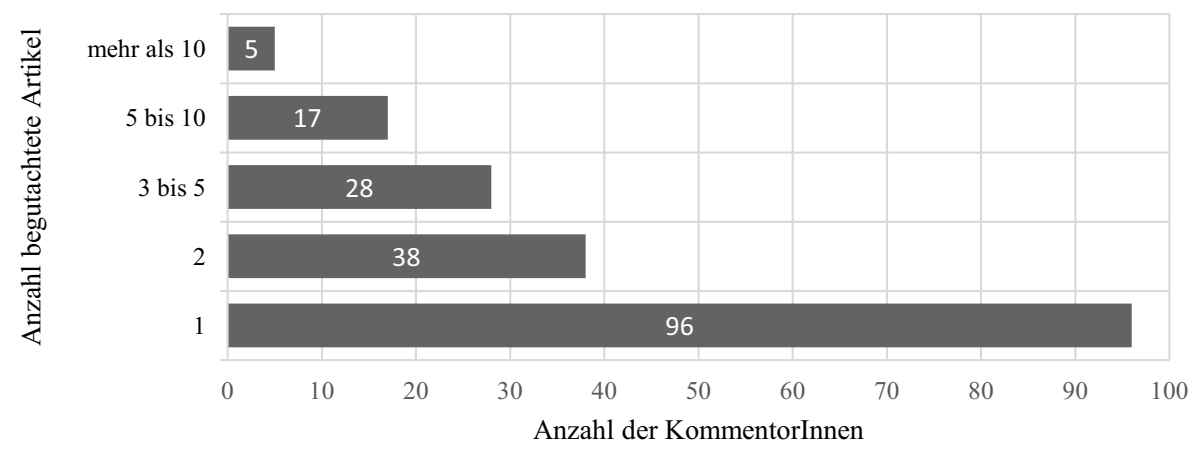

Abb. 3 Anzahl an KommentatorInnen und begutachtete Artikel

\section{Literatur}

Blöbaum, B. (1994). Journalismus als soziales System. Geschichte, Ausdifferenzierung und Verselbständigung. Wiesbaden: Springer.

Blöbaum, B. (2016). Journalismus als Funktionssystem der Gesellschaft. In M. Löffelholz \& L. Rothenberger (Hrsg.), Handbuch Journalismustheorien (S. 151-163). Wiesbaden: Springer.

Blöbaum, B. (2017). Wissenschaftsjournalismus. In H. Bonfadelli, B. Fähnrich, C. Lüthje, J. Milde, M. Rhomberg \& M.S. Schäfer (Hrsg.), Forschungsfeld Wissenschafts-kommunikation (S. 221-238). Wiesbaden: Springer.

Bray, D., \& von Storch, H. (2007). The perspectives of climate scientists on global climate change. Geesthacht: GKSS-Forschungszentrum Geesthacht $\mathrm{GmbH}$.

Brüggemann, M., \& Engesser, S. (2017). Beyond false balance: How interpretive journalism shapes media coverage of climate change. Global Environmental Change, 42, 58-67.

Brüggemann, M., Neverla, I., Hoppe, I., \& Walter, S. (2018). Klimawandel in den Medien. In H. von Storch, I. Meinke \& M. Claußen (Hrsg.), Hamburger Klimabericht - Wissen über Klima, Klimawandel und Auswirkungen in Hamburg und Norddeutschland (S. 243-254). Berlin: Springer.

Brüggemann, M., Lörcher, I., \& Walter, S. (2020). Post-normal science communication: exploring the blurring boundaries of science and journalism. Journal of Science Communication, 19(03), A2.

Climate Feedback (2015). Analysis of "2014 Breaks Heat Record, Challenging Global Warming Skeptics". https://climatefeedback.org/evaluation/new-york-times-justin-gillis-2014-breaks-heat-record/. Zugegriffen: 14. Apr. 2018.

Climate Feedback (o.J. a). Community. https://climatefeedback.org/community/. Zugegriffen: 14. Juli 2019.

Climate Feedback (o.J. b). About. http://climatefeedback.org/about/. Zugegriffen: 14. April 2018.

Climate Feedback (o.J. c). Partners, Funders \& Donors. https://cimatefeedback.org/partners-fundersdonors/. Zugegriffen: 14. April 2018.

Deutsche Welle (2018). Climate change: 'Fake news,' real fallout. https://www.dw.com/en/climatechange-fake-news-real-fallout/a-44603523. Zugegriffen: 13. Juli 2019.

Dudo, A. (2015). Scientists, the media, and the public communication of science. Sociology Compass, 9 , $761-775$.

Dunlap, R.E., \& McCright, A.M. (2015). Challenging climate change: the denial countermovement. In R.E. Dunlap \& R.J. Brulle (Hrsg.), Climate change and society. Sociological perspectives (S. 300-332). New York: Oxford University Press.

Eide, E., \& Kunelius, R. (2012). Introduction. In E. Eide \& R. Kunelius (Hrsg.), Media meets climate: the global challenge for journalism (S. 9-30). Göteborg: Nordicom.

Fähnrich, B., \& Lüthje, C. (2017). Roles of social scientists in crisis media reporting: the case of the German populist radical right movement PEGIDA. Science Communication, 39, 415-442.

Farnsworth, S. J., \& Lichter, S. R. (2012). Scientific assessments of climate change information in news and entertainment media. Science Communication, 34, 435-459. 
Franzen, M., Weingart, P., \& Rödder, S. (2012). Exploring the impact of science communication on scientific knowledge production: an introduction. In S. Rödder, M. Franzen \& P. Weingart (Hrsg.), The sciences' media connection-public communication and its repercussions (S. 3-14). Dordrecht: Springer.

Funtowicz, S. O., \& Ravetz, J. R. (1993). Science for the post-normal age. Futures, 25, 739-755.

Hutter, A. (2009). Watchblogs: Medienkritik 2.0? Eine inhaltsanalytische Untersuchung journalistischer Qualität in medienkritischen Weblogs. Boizenburg: Werner Hülsbusch.

Independent (Hrsg.). (2017). Climate change doomsday warning of 'rolling death smog' and 'perpetual war' criticised by scientists. https://www.independent.co.uk/environment/climate-changedoomsday-scenario-new-york-magazine-perpetual-war-rollnig-death-smog-critics-a7838991.html. Zugegriffen: 14. Juli 2019.

Ivanova, A., Schäfer, M.S., Schlichting, I., \& Schmidt, A. (2013). Is there a medialization of climate science? Results from a survey of German climate scientists. Science Communication, 35, 626-653.

Kohring, M. (1997). Die Funktion des Wissenschaftsjournalismus. Ein system-theoretischer Entwurf. Opladen: Westdeutscher Verlag.

Kohring, M. (2016). Journalismus als Leistungssystem der Öffentlichkeit. In M. Löffelholz \& L. Rothenberger (Hrsg.), Handbuch Journalismustheorien (S. 165-176). Wiesbaden: Springer VS.

Krause, D. (2005). Luhmann-Lexikon: Eine Einführung in das Gesamtwerk von Niklas Luhmann; mit über 600 Lexikoneinträgen einschließlich detaillierter Quellenangaben (4. Aufl.). Stuttgart: Lucius \& Lucius.

Landis, J. R. \& Koch, G. G. (1977). The measurement of observer agreement for categorical data. Biometrics, 33, 159-174.

Lehmkuhl, M., \& Leidecker-Sandmann, M. (2019). „Visible Scientists Revisited“: Zum Zusammenhang von wissenschaftlicher Reputation und der Präsenz wissenschaftlicher Experten in der Medienberichterstattung über Infektionskrankheiten. Publizistik, 64, 479-502.

Loosen, W. (2016). Journalismus als (ent-)differenziertes Phänomen. In M. Löffelholz \& L. Rothenberger (Hrsg.), Handbuch Journalismustheorien (S. 177-189). Wiesbaden: Springer VS.

Luhmann, N. (1996). Die Realität der Massenmedien (2. Aufl.). Opladen: Westdeutscher Verlag.

McKnight, D. (2010). A change in the climate? The journalism of opinion at news corporation. Journalism, $11,693-706$.

Meier, K. (2007). Journalistik. Konstanz: UVK.

Neverla, I., \& Schäfer, M. S. (2012). Einleitung: Der Klimawandel und das „Medien-Klima“. In I. Neverla \& M.S. Schäfer (Hrsg.), Das Medien-Klima. Fragen und Befunde der kommunikationswissenschaftlichen Klimaforschung (S. 9-25). Wiesbaden: Springer.

Painter, J. (2013). Climate change in the media: reporting risk and uncertainty. London: Tauris.

Peters, H. P., Dunwoody, S., Allgaier, J., Lo, Y.-Y., \& Brossard, D. (2014). Public communication of science 2.0. EMBO Reports, 15(7), 749-753.

Post, S. (2008). Klimakatastrophe oder Katastrophenklima? Die Berichterstattung über den Klimawandel aus Sicht der Klimaforscher. München: Verlag Reinhard Fischer.

Post, S. (2013). Wahrheitskriterien von Journalisten und Wissenschaftlern. Baden-Baden: Nomos.

Post, S. (2016). Communicating science in public controversies: strategic considerations of the German climate scientists. Public Understanding of Science, 25, 61-70.

Rahmstorf, S., \& Schellnhuber, H.J. (2007). Der Klimawandel. Diagnose, Prognose, Therapie. Bd. 5. München: C.H. Beck.

Rödder, S. (2009). Reassessing the concept of a medialization of science: a story from the "book of life". Public Understanding of Science, 18, 452-463.

Rühl, M. (1980). Journalismus und Gesellschaft. Bestandsaufnahme und Theorieentwurf. Mainz: v. Hase Koehler.

Schäfer, M.S., Kristiansen, S., \& Bonfadelli, H. (2015). Wissenschaftskommunikation im Wandel: Relevanz, Entwicklungen und Herausforderungen des Forschungsfeldes. In M. S. Schäfer, S. Kristiansen \& H.H. Bonfadelli (Hrsg.), Wissenschaftskommunikation im Wandel (S. 10-42). Köln: Herbert von Halem.

Scheloske, M. (2012). Bloggende Wissenschaftler - Pioniere der Wissenschaftskommunikation 2.0. In B. Dernbach, C. Kleinert \& H. Münder (Hrsg.), Handbuch Wissenschaftskommunikation (S. 267-274). Wiesbaden: Springer.

Schimank, U. (2002). Handeln und Strukturen. Einführung in die akteurtheoretische Soziologie (2. Aufl.). Weinheim: Juventa.

Schimank, U. (2007). Handeln in Konstellationen: Die reflexive Konstitution von handelndem Zusammenwirken und sozialen Strukturen. In K.-D. Altmeppen, T. Hanitzsch \& C. Schlüter (Hrsg.), Journalis- 
mustheorie: Next Generation. Soziologische Grundlegung und theoretische Innovation (S. 121-137). Wiesbaden: VS.

Scholl, A., \& Weischenberg, S. (1998). Journalismus in der Gesellschaft. Theorie, Methodologie und Empirie. Opladen: Westdeutscher Verlag.

Schreier, M. (2012). Qualitative content analysis in practice. London: SAGE.

The Guardian (Hrsg.). (2017). Fake news is a threat to humanity, but scientists may have a solution. https://www.theguardian.com/environment/climate-consensus-97-per-cent/2017/dec/27/fake-newsis-a-threat-to-humanity-but-scientists-may-have-a-solution. Zugegriffen: 14. Juli 2019.

The Huffington Post (Hrsg.). (2017). New York times readers are canceling subscriptions over climatedenying writer. https://www.huffpost.com/entry/new-york-times-bret-stephens-subscriptions_n_ 5904171ce4b02655f83db230?guccounter=1. Zugegriffen: 14. Juli 2019.

Walter, S., \& Brüggemann, M. (2020). Opportunity makes opinion leaders: analyzing the role of first-hand information in opinion leadership in social media networks. Information, Communication \& Society, 23, 267-287.

Walter, S., Lörcher, I., \& Brüggemann, M. (2019). Scientific networks on twitter: analyzing scientists' interactions in the climate change debate. Public Understanding of Science, 28, 696-712.

Weingart, P. (2001). Die Stunde der Wahrheit? Zum Verhältnis der Wissenschaft zu Politik, Wirtschaft und Medien in der Wissensgesellschaft. Weilerswist: Velbrück.

Wied, K., \& Schmidt, J. (2008). Weblogs und Qualitätssicherung. Zu Potenzialen weblogbasierter Kritik im Journalismus. In T. Quandt \& W. Schweiger (Hrsg.), Journalismus online - Partizipation oder Profession? (S. 173-192). Wiesbaden: VS.

Wormer, H. (2017). Vom public understanding of science zum public understanding of journalism. In H. Bonfadelli, B. Fähnrich, C. Lüthje, J. Milde, M. Rhomber \& M. S. Schäfer (Hrsg.), Forschungsfeld Wissenschaftskommunikation (S. 429-451). Wiesbaden: Springer.

Stefanie Walter ist Post-Doc am Zentrum für Medien-, Kommunikations- und Informationsforschung (ZeMKI) der Universität Bremen.

Janne Görlach ist Pressereferentin bei Agora Energiewende in Berlin. Bis 2018 war sie Masterstudentin an der Universität Hamburg.

Prof. Dr. Michael Brüggemann ist Inhaber des Lehrstuhls für Kommunikationswissenschaft, insbesondere Klima- und Wissenschaftskommunikation an der Universität Hamburg. 